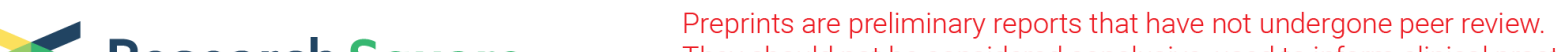 Research Square They should not be considered conclusive, used to inform clinical practice, or referenced by the media as validated information.
}

\section{Paraneoplastic Cerebellar Degeneration Associated With anti-Yo Antibodies Appearing as a Leptomeningeal Cerebellar Carcinomatosis at Mri. A Case Report.}

\section{Massimo Napolitano ( $\square$ max990@libero.it)}

Azienda Ospedaliera di Rilievo Nazionale Antonio Cardarelli https://orcid.org/0000-0003-1853-2737

\section{Angelo Ranieri}

AORN Cardarelli: Azienda Ospedaliera di Rilievo Nazionale Antonio Cardarelli

\section{Gennaro Alfieri}

AORN Cardarelli: Azienda Ospedaliera di Rilievo Nazionale Antonio Cardarelli

\section{Giorgia Teresa Maniscalco}

AORN Cardarelli: Azienda Ospedaliera di Rilievo Nazionale Antonio Cardarelli

\section{Fabio Zeccolini}

AORN Cardarelli: Azienda Ospedaliera di Rilievo Nazionale Antonio Cardarelli

\section{Amedeo Boscaino}

AORN Cardarelli: Azienda Ospedaliera di Rilievo Nazionale Antonio Cardarelli

\section{Valentino Manzo}

AORN Cardarelli: Azienda Ospedaliera di Rilievo Nazionale Antonio Cardarelli

\section{Case Report}

Keywords: Paraneoplastic, Degeneration, Carcinomatosis

Posted Date: June 4th, 2021

DOI: https://doi.org/10.21203/rs.3.rs-422509/v1

License: (c) (i) This work is licensed under a Creative Commons Attribution 4.0 International License. Read Full License 


\section{Abstract}

Anti Yo antibodies (AYA) are the antibodies most frequently identified with Paraneoplastic Cerebellar Degeneration (PCD). They are detected above all in PCD associated with breast and gynecologic malignancies. Here, we report a third case of PCD with cerebellar contrast enhancement findings at MRI. In fact, AYA cross anti cerebellar cortex immune reaction leads mainly to hyperintensity signals in T2weighted-MRI sequences, followed by cerebellar atrophy. As such, meningeal contrast enhancement could lead to a diagnostic pitfall as it can be considered a carcinomatosis - above all in serum antibody negative cases as well as when a metastatic tumour is found. Our patient had no tumoral cells in the cerebrospinal fluid and her neurological syndrome remained stable after immunoglobulin treatment. AYA serum finding was diriment for PCD diagnosis.

\section{Introduction}

Paraneoplastic Cerebellar Degeneration (PCD) is a rare syndrome characterized by cerebellar dysfunction, typically with a subacute onset, associated with the presence of specific onconeural auto-antibodies (aAb). Particularly, serum detection of anti-Yo aAb strongly supports the paraneoplastic etiology of cerebellar disease [1]. Indeed, the vast majority of patients with anti-Yo antibodies (AYA) have a breast or gynaecological cancer that develops over months to years after the neurological onset of symptoms. Sometimes Hodgkin's lymphoma, or a small-cell lung cancer, can also be found. Many patients become disabled because of a severe progressive ataxia. In early stages, MRI imaging is often normal, although in some cases a cerebellar FLAIR hyperintensity has been reported [1-3].Cerebellar atrophy is the main neuroradiological finding and is usually observed later in the course of the disease. Contrast enhancement of the cerebellar cortex is very rare, having been described only in a patient with nonHodgkin's lymphoma and PCD with anti-Tr antibodies [4]. More recently, a case of nasopharyngeal carcinoma-associated anti-Yo-PCD showed a transient diffuse leptomeningeal enhancement on MRI imaging [5]. We present an unusual case of PCD with enhancing cerebellar lesions, leading to a possible diagnosis of cerebral carcinomatosis.

\section{Case Presentation}

A 53 year- with a subacute onset of ataxia and dysarthria, which had lasted for ca 4 weeks, was hospitalised in our department. She also described having double vision for a brief period, however this had been resolved at time of admission. The neurological examination showed an alert and oriented patient with severe trunk ataxia, dysarthria, horizontal nystagmus, dysmetria and intention tremor. The patient's medical history was unremarkable except for a previous Hashimoto thyroiditis. Brain MRI showed a meningeal cerebellar intensive contrast enhancement, associated with micro nodulations of the cerebellar hemisphere cortex (Fig. 1). Initially, the findings were concerning due to the possibility of a neoplastic process, such as a brain carcinomatosis in the structures of posterior cranial fossa versus an inflammatory cerebellitis. However, cerebral spinal fluid (CSF) analysis on two different samples performed two weeks apart did not reveal malignant cells. On the contrary, some inflammatory changes 
were detected on the first CSF analysis: mild pleocytosis $(21 \mathrm{cells} / \mathrm{mmc})$, high proteins $(73 \mathrm{mg} / \mathrm{dl})$ and oligoclonal bands. We looked for and found anti-Yo antibodies in the serum, fulfilling the diagnostic criteria for definite Paraneoplastic Neurological Syndrome (PNS) [6]. A body CT scan was then performed, without detecting a clear primitive cancer. Mammography was not done as the patient could not stand; however her breast ultrasound was unremarkable. Intravaginal ultrasound study revealed an intramural uterine nodule and a left ovarian structural alteration. There was a mild increase of CA-125 oncomarker. The patient underwent treatment with a high dose of intravenous steroids (methylprednisolone $1 \mathrm{gr}$ for 5 days), without clinical changes, followed by intravenous IgG treatment $(0,4 \mathrm{gr} / \mathrm{kg}$ in 5 days) obtaining a slight improvement of symptoms. A Brain MRI, performed 10 days after the latter treatment, also showed a positive evolution. Indeed, no cerebellar contrast enhancement lesions were present, whereas a mild cerebellar atrophy was noticed (Fig. 1). In order to continue the research of the underlying tumor, we performed an 18-FDG PET/CT, showing a single spot hypermetabolism in the right inguinal region. A lymph node was then detected and removed. The histological examination revealed an undifferentiated cancer of possible ovarian origin based on immunohistochemical analysis (Fig. 2). The patient was advised to have a surgical treatment of ovariectomy with the intention of achieving maximum recovery of the neurological syndrome. Unfortunately she refused the surgery and her neurological condition was unchanged 6 months after the onset.

\section{Discussion}

Our patient showed cerebellar contrast enhanced lesions at MRI imaging that could lead to a diagnosis of cerebellar leptomeningeal carcinomatosis. A viral cerebellitis could also have been considered. However, while a cerebellar syndrome was observed, typical clinical signs and symptoms of carcinomatosis like headache, cranial nerve palsy, spine pains or disturbances in consciousness were absent. Moreover no neoplastic cells were revealed on two different CSF samples. As such, until the positive response in the serum of anti-Yo $a A b$, we remained very doubtful about a PCD diagnosis. Indeed, brain MRI, in most cases, is normal or shows few abnormalities in T2-Flair weighted sequences at the onset of disease which is followed by atrophy in later stages, while post-contrast enhanced lesions are anecdotal [4-5]. At this point however, our reservations around the diagnosis were dissolved as we did not notice an unfavourable clinical course. Moreover, a few days after the immunosuppressive treatment no enhancing lesions at MRI were seen. Paraneoplastic neurological syndromes can sometimes mimic a neoplasm because the inflammatory autoimmune reaction can be very intense, leading to diffuse edema and damage in the blood brain barrier [7]. Immunosuppressant therapy could have an active role in reducing this pathological response. We suggest that onconeural antibody serum research is mandatory in an attempt to differentiate some atypical MRI findings in neurological isolated syndromes of uncertain origin.

\section{Declarations}

Ethics \& Declarations 
Funding

There was no funding for this report

Conflict of Interest

The authors declare that they have no conflict of interest.

Ethical approval

Ethical approval was not required in this case.

Informed consent

Written informed consent was obtained from the patient included in the case report

MN wrote the manuscript. MN and AR conceived the case report. AR, GTM, GA and VM analyzed the data.

FZ described MRI findings and selected the radiological images. AB made histopathological analysis and choice the images. All authors read and approved the manuscript

\section{References}

1. Peterson K, Rosenblum MK, Kotanides H, Posner JB Paraneoplastic cerebellar degeneration. I. A clinical analysis of 55 anti-Yo antibody-positive patients. Neurology 4 (1992) 1931-7

2. Gilmore CP, Elliott I, Auer D, Maddison P Diffuse cerebellar MR imaging changes in anti-Yo positive paraneoplastic cerebellar degeneration. J Neurol 257 (2010) 490-1

1. McHugh JC, Tubridy N, Collins CD, Hutchinson M Unusual MRI abnormalities in anti-Yo positive "pure" paraneoplastic cerebellar degeneration. J Neurol 255 (2008) 138-9

2. Josep Dalmau and Myrna R Rosenfeld. Paraneoplastic syndromes of the CNS. Lancet Neurol. Apr 7(4): 2008 327-340.

3. S Bhardawaj, S. Khasani, D. Benasher et al . Paraneoplastic cerebellar degeneration in nasopharyngeal carcinoma: a Unique Association. The Cerebellum 18, (2019) 1126-1129

4. Graus F, Delattre JY, Antoine JC, et al Recommended diagnostic criteria for paraneoplastic neurological syndromes. J Neurol Neurosurg Psychiatry 75: (2004) 1135-40

5. A.A. Madhavan, C.M. Carr, P.P. Morris, E.P. Flanagan, A.L. Kotsenas, C.H. Hunt, L.J. Eckel, E.P. Lindell, and F.E. Diehn Imaging Review of Paraneoplastic Neurologic Syndromes AJNR Am J Neuroradiol: $2020 \mathrm{w}$

\section{Figures}



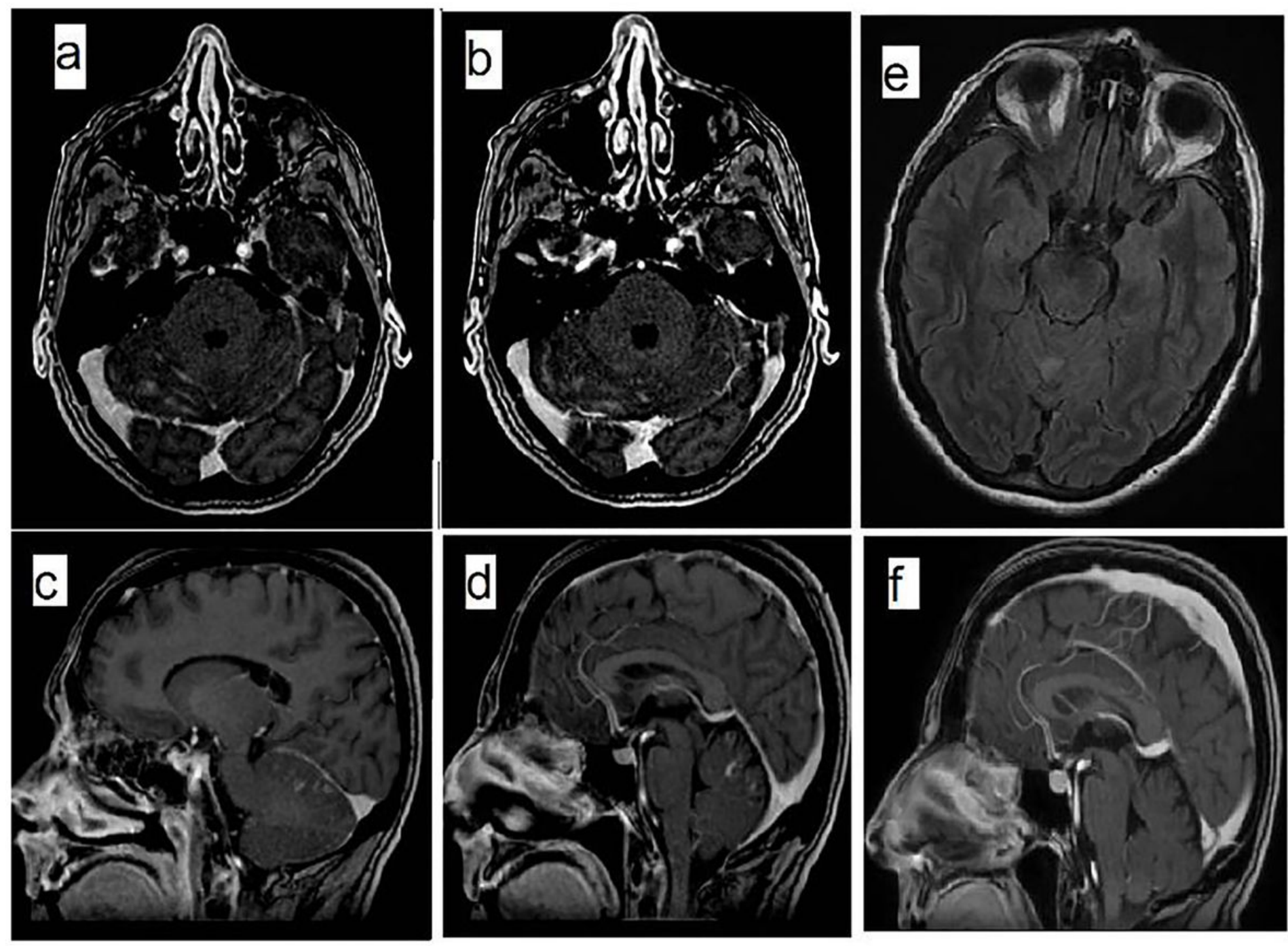

Figure 1

Brain MRI at the onset $(a, b, c, d, e)$ : axial $(a, b)$ and sagittal $(c, d)$ T1-weighted contrast images showed a slight, irregular cortical and meningeal enhancement in both upper cerebellar hemispheres, suggesting a possible meningeal carcinomatosis; axial FLAIR-weighted image (e) showed the hyperintense signal of the superior cerebellar vermis and upper cerebellar hemispheres. Brain MRI after immunosuppressive treatment at 1 month (f): the contrasted images sequences revealed no contrast enhancement and initial cerebellar atrophy. 


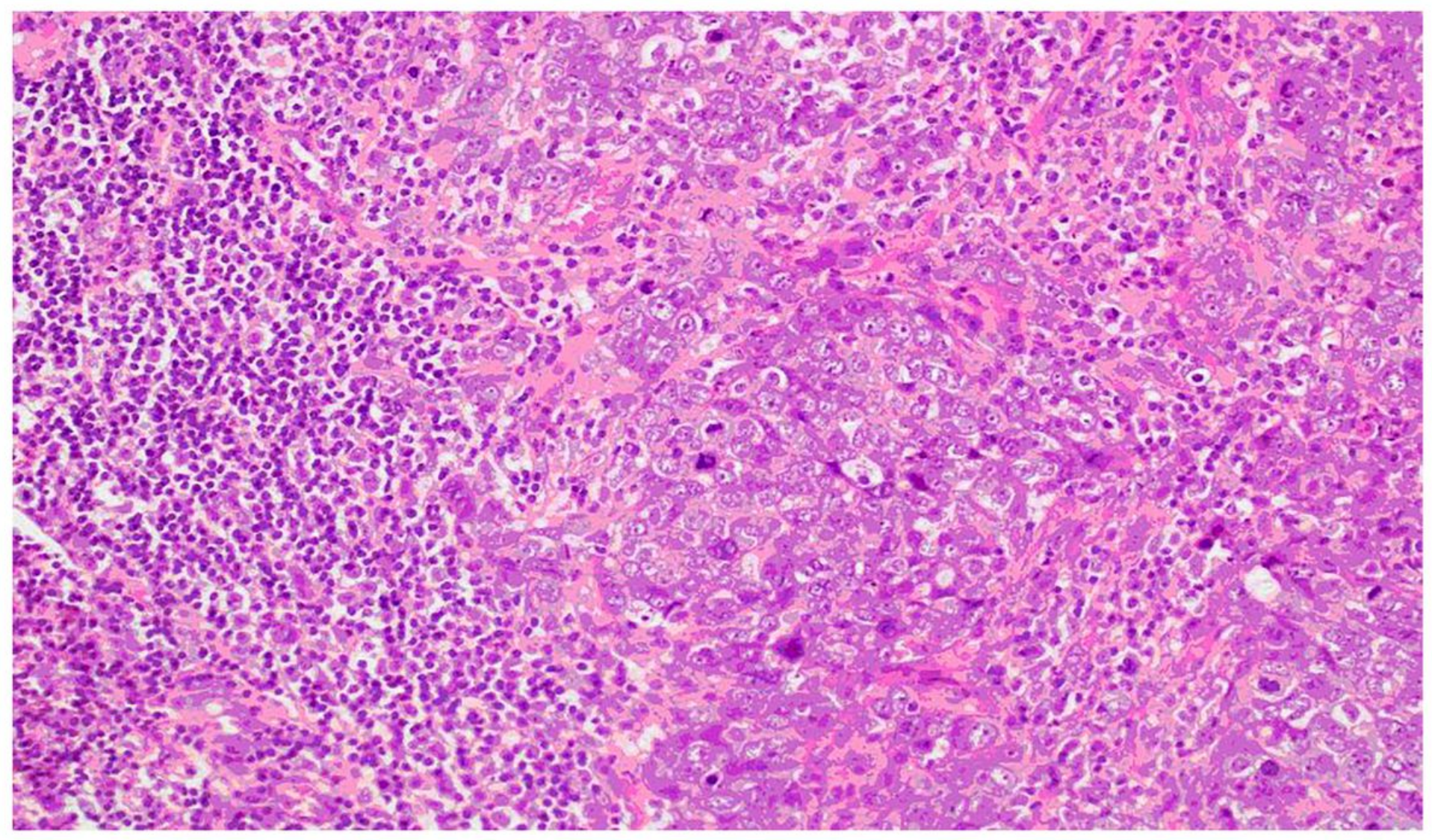

\section{Figure 2}

Hematoxylin-eosin staining of the excised lymph node shows a widespread metastasis from poorly differentiated carcinoma. Immunohistochemical profile on neoplastic cells resulted positive for Cytokeratin 7, PAX8, focally for P40; WT1 estrogen receptor (about 90\%) and weak and focal positive for progesterone receptors (10\%). Neoplastic cells resulted negative for P63, TTF1, Cytokeratin 20, GATA 3, Synaptophysin. Conclusion: Lymph node metastasis from high grade adenocarcinoma (G3), possible genital origin (ovarian-uterine cavity) serous adenocarcinoma. 